\title{
A view of the sub-mJy populations, modelling and perspectives for future deep surveys
}

\section{Isabella Prandoni*, Paola Parma}

INAF - Istituto di Radioastronomia, Via Gobetti 101, Bologna (Italy)

E-mail: prandonieira.inaf.it

\section{Arturo Mignano}

INAF - Oss. Astronomico di Brera, Via Brera 28, Milano (Italy)

\section{Hans R. de Ruiter}

INAF - Oss. Astronomico di Bologna, Via Ranzani 1, Bologna (Italy)

\section{Loretta Gregorini}

Dip. Fisica, Univ. degli studi di Bologna, Via Irnerio 46, Bologna (Italy)

\section{Giampaolo Vettolani}

INAF, Viale del Parco Mellini 84, Roma (Italy)

\section{Mark H. Wieringa and Ron D. Ekers}

CSIRO Australia Telescope Facility, PO Box 76, Epping NSW 2121 (Australia)

We use deep multi-colour (UBVRIJK) images mostly taken in the framework of the ESO Deep Public Survey (DPS) to optically identify and derive photometric redshifts for a complete sample of 131 radio sources with $S>0.4 \mathrm{mJy}$, observed at both 1.4 and $5 \mathrm{GHz}$ as part of the ATESP radio survey. The availability of multi-wavelength radio and optical information is exploited to infer the physical properties of the faint radio population. In particular we find that, considering both early-type galaxies and quasars as sources with an active nucleus, AGNs largely dominate our sample sub-mJy sample (78\%). Further radio/optical analysis of such AGN component has revealed a somewhat unexpected class of flat/inverted-spectrum sources with low radio-to-optical ratios $(R<100)$, which are preferentially identified with early-type galaxies. Such sources are quite compact $(d<10-30 \mathrm{kpc})$, suggesting core-dominated radio emission triggered by low luminosity AGNs. This intriguing class of objects deserves further analysis, and new higher resolution radio observations are currently under way. In parallel we are developing radio source models, for both the AGN and the star-forming components of the sub-mJy radio pupulation. Here we discuss the first results.

From Planets to Dark Energy: the Modern Radio Universe October 1-5 2007

The University of Manchester, UK

\footnotetext{
* Speaker.
} 


\section{Scientific Background}

One of the most debated issues about the sub-milliJy (sub-mJy) radio sources responsible for the steepening of the $1.4 \mathrm{GHz}$ source counts ([2], [19]) is the origin of their radio emission. There are strong theoretical and observational reasons, based also on studies at other wavelengths, to believe that such sources are mainly rapidly evolving star-forming galaxies, but there is also observational evidence that the contribution of other classes of objects in the radio below $1 \mathrm{mJy}$ is important. Multi-wavelength studies of deep radio fields show that star-forming galaxies dominate the microJy ( $\mu \mathrm{Jy}$ ) population (see e.g. [17]) while early-type galaxies and AGN become dominant at flux densities $>0.1-0.2 \mathrm{mJy}$ (see e.g. [6], [9], [15], [1], [11]). Large numbers of so-called radio-intermediate quasars have been found at mJy levels (see e.g. [8]) and Jarvis \& Rawlings ([7]) propose a scenario in which radio-quiet quasars strongly affect the radio counts in the flux range $0.3-1$ mJy. However, the picture outlined above needs further confirmation since most efforts so far have been devoted to the study of $\mu \mathrm{Jy}$ samples, where star-forming galaxies dominate.

Assessing whether the AGN-triggered component of the sub-mJy population is more related to efficiently accreting systems - like radio-intermediate/quiet quasars - or to systems with very low accretion rates - like e.g. FRI ([4]) radio galaxies - would have a relevant impact on the study of the physical and evolutionary properties of low-power radio AGNs, and, more generally, would allow us a better understanding of the triggering mechanisms of AGN radio activity.

\section{Observational Results: the ATESP-DEEP1 Sample}

One especially suited radio sample to study the phenomenon of low-luminosity nuclear activity, possibly related to low efficiency accretion processes and/or radio-intermediate/quiet QSOs, is the so-called ATESP-DEEP1 sample, which consists of 131 radio sources with $S_{\text {lim }} \sim 0.4 \mathrm{mJy}$, covering a one square degree area and imaged at both 5 and $1.4 \mathrm{GHz}$ in the framework of the Australia Telescope ESO Slice Project (ATESP) survey (see [13], [14], [16]). It is worth to note that at the flux densities probed here (mainly $0.5-1 \mathrm{mJy}$ ) star-forming galaxies should start to contribute but should not yet be the dominant population and, in addition, this sub-mJy sample is one of the few with multi-frequency radio information. Deep $(I<24)$ UBVRIJK multi-color imaging is also available from the ESO Deep Public Survey (DPS, see [10], [12]), allowing to identify and get a redshift determination for about $80 \%$ of the radio sources (see [11]). We confirm that at the submJy level the large majority of sources are associated with objects that have early-type (64\%) and AGNs (14\%) spectra. Although earlier work (based on shallower optical follow-up) revealed the presence of a conspicuous component of late-type and star-burst objects, such objects appear to be important only at bright magnitudes $(I<19)$, and are rare at fainter magnitudes.

From an overall comparison of the radio spectral index with other radio and optical properties of the ATESP-DEEP1 sample, we find that most sources with flat radio spectra have high radio-tooptical ratios, as expected for classical radio galaxies and quasars, while star-forming galaxies are associated to steep-spectrum radio sources. However the multi-frequency/multi-band analysis has revealed a somewhat unexpected class of objects with flat and/or inverted spectrum and low radioto-optical ratios $(10<R<1000$, see Fig. 1, top panel). Such sources are compact, with linear sizes $d<10-30 \mathrm{kpc}$ (see Fig. 1, middle panel), and associated to optically inactive early-type galaxies 


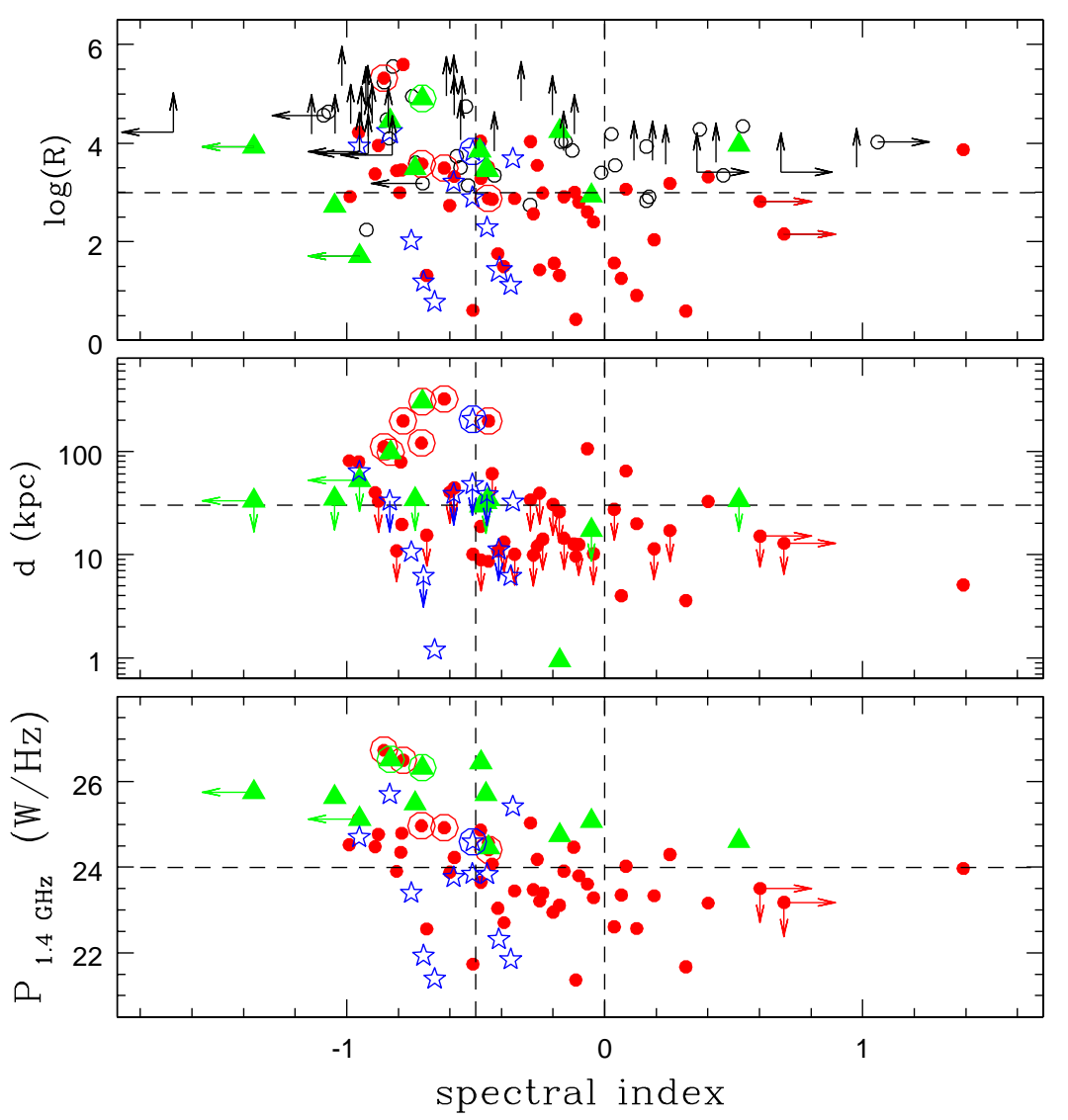

Figure 1: Radio-to-optical ratio ( $R$, top panel), linear radio source size ( $d$ in kpc, middle panel) and 1.4 $\mathrm{GHz}$ radio power (in $\mathrm{W} / \mathrm{Hz}$, bottom panel) against $1.4-5 \mathrm{GHz}$ spectral index for the ATESP-DEEP1 radio sources: red filled circles (ETS); blue stars (LTS/SB); green filled triangles (AGNs). Circled symbols indicate sources with extended and/or two-component radio morphology, typical of classical radio galaxies. Black open circles are for identified sources which do not have a redshift/type determination. Arrows indicate upper/lower limits. Vertical dashed lines indicate the $\alpha=-0.5$ and the $\alpha=0$ values, above which source spectra are defined respectively as flat and inverted. Horizontal dashed lines in the three panels indicate respectively (from top to bottom) values of $R=1000, d=30 \mathrm{kpc}$ and $P_{1.4 \mathrm{GHz}}=10^{24} \mathrm{~W} / \mathrm{Hz}$.

(see [11]). The distribution in redshift of these galaxies extends up to $\mathrm{z} \sim 2$, showing a significant peak at $\mathrm{z}=0.5$, indicating that such sources may undergo significant evolution.

Their radio luminosities $\left(P_{1.4 \mathrm{GHz}} \sim 10^{22-24} \mathrm{WHz}^{-1}\right.$, see Fig. 1, bottom panel) and the absence of emission lines in their optical spectra may suggest that these objects belong to the class of FRI radio galaxies. FRI radio galaxies, however, are characterized on average by steeper radio spectra and larger linear sizes. The compactness of the sources, together with the flat/inverted spectra, suggests core emission with strong synchrotron or free-free self-absorption. Such sources may therefore either represent a specific sub-class of core-dominated FRI radio galaxies, or may be associated to specific phases in the life of a radio source, or also may be similar to the low power compact radio sources discussed by Giroletti et al. ([5]). 


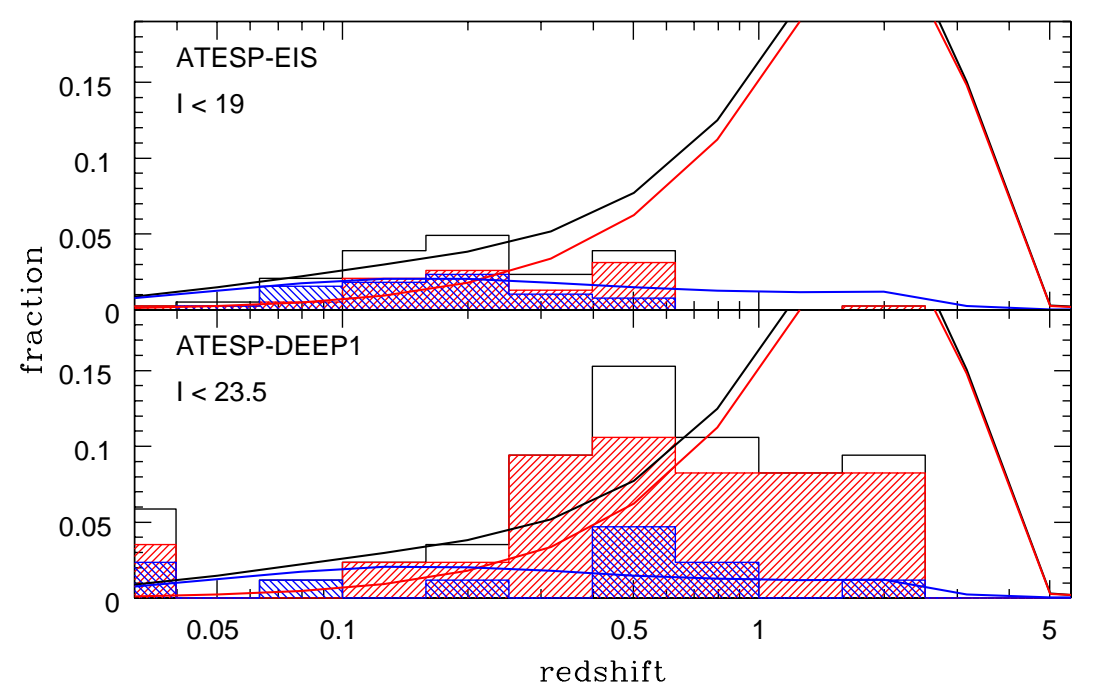

Figure 2: Redshift distribution for the shallower $(I<19)$ ATESP-EIS sample (top panel) and for the deeper $(I<24)$ ATESP-DEEP1 sample (bottom panel). The observed redshift distributions (black histograms) are compared to the modeled ones (black lines). Red lines and histograms indicate the AGN fraction (steep + flat components); Blue lines and histograms indicate the star-forming galaxy fraction (evolving + non-evolving components).

\section{Comparison with Models}

In order to probe the evolution of low-luminosity radio AGNs we have compared our observational results with standard models developed for the faint radio population. We have modeled the AGN component following Dunlop \& Peacock ([3]) in two sub-components: steep and flat AGNs. The star-forming galaxies have been modeled starting from the local radio luminosity function of Sadler et al. ([18]) in two sub-components: non-evolving normal spirals and evolving starburst galaxies $\left(L \sim(1+z)^{3}\right)$.

In Fig. 2 we compare the models to the source redshift distribution of the shallower $(I<19)$ ATESP-EIS 3 sq. degr. sample (studied in [15], top panel) and of the deeper $(I<24)$ ATESPDEEP1 sample (bottom panel). The Figure clearly shows that only going to deeper optical magnitudes we can really probe the models developed for the faint radio population and that standard modeling can reproduce with good accuracy the redshift distribution of the ATESP-DEEP1 sample, for both the AGN and star-forming galaxy components.

\section{Conclusions and Future Perspectives}

In this paper we have discussed the nature of the sub-mJy radio population, through the radio/optical analysis of the ATESP-DEEP1 sample. The high percentage of identifications $(\sim 78 \%)$ allowed us to make a direct comparison between observations and models, in order to assess the nature and the evolutionary properties of the sub-mJy population, with particular respect to the AGN 
component. Our radio/optical analysis has revealed a class of flat/inverted spectrum radio sources associated to optically inactive early-type galaxies, which are most plausibly triggered by lowaccretion/radiative efficiency AGNs. In order to further investigating this class of objects higher resolution multi-frequency radio observations are on-going.

We find a general agreement between observations and standard pure luminosity evolution radio source modeling. It is clear however that, to provide more quantative constraints to the models, we need larger deep radio samples together with complete optical follow-up. In particular deeper radio surveys are necessary to probe a possible radio-quiet AGN component, which does not show up at the flux densities probed by our sample $(S>0.4 \mathrm{mJy})$.

A clear understanding of the nature and evolutionary properties of the various components of the sub-mJy population is a very important issue for providing reliable modeling of the nanoJy radio sky will be eventually probed by the SKA, and will be importat matter of study for the new upcoming radio facilities (e.g. LOFAR, EVLA, eMERLIN, etc.).

\section{References}

[1] Afonso J., Mobasher B., Koekemoer A., Norris R.P., Cram L., 2006, AJ, 131, 1216

[2] Condon J.J., 1984, ApJ, 287, 461

[3] Dunlop J.S. \& Peacock J.A., 1990, MNRAS, 247, 19

[4] Fanaroff B.L., Riley J.M., 1974, MNRAS, 167P, 31F

[5] Giroletti M., Giovannini G., Taylor G.B., 2005, A\&A, 441, 89

[6] Gruppioni C., Ciliegi P., Rowan-Robinson M., et al., 1999, MNRAS 305, 297

[7] Jarvis M.J. \& Rawlings S., 2004, New Astron. Rev., 48, 1173

[8] Lacy M., Laurent-Muehleisen S.A., Ridgway S.E., Becker R.H., White R.L., 2001, ApJ, 551, L17

[9] Magliocchetti M., Maddox S.J., Wall J.V., et al., 2000, MNRAS, 318, 1047

[10] Mignano A., Miralles J.-M., da Costa L., et al., 2007a, A\&A, 462, 553

[11] Mignano A., Prandoni I., Gregorini L., et al. 2007b, A\&A, in press, arXiv:0710.5682

[12] Olsen L.F., Miralles J.-M., da Costa L., et al., 2006, A\&A, 456, 881

[13] Prandoni I., Gregorini L., Parma P., et al., 2000a, A\&AS, 146, 31

[14] Prandoni I., Gregorini L., Parma P., et al., 2000b, A\&AS, 146, 41

[15] Prandoni I., Gregorini L., Parma P., et al., 2001, A\&A, 369, 787

[16] Prandoni I., Parma P., Wieringa M.H., et al., 2006, A\&A, 457, 517

[17] Richards E.A., Fomalont E.B., Kellermann K.I., et al., 1999, ApJ 526, L73

[18] Sadler E.M., Jackson C.A., Cannon R.D., et al., 2002, MNRAS, 329, 227

[19] Windhorst R.A., Mathis D., Neuschaefer L., 1990. In: Kron R.G. (ed.) Evolution of the Universe of Galaxies, ASP Conf. Ser. 10, 389 